\title{
On Reachability and Stabilization of Switched Linear Systems*
}

\author{
Zhendong Sun $^{\dagger}$ and Dazhong Zheng \\ Department of Automation, Tsinghua University \\ Beijing 100084, P.R. China
}

\begin{abstract}
In this paper, the reachability and stabilization issues are addressed for switched linear control systems. A necessary condition and a sufficient condition are presented for reachability. Under mild assumptions, we prove that the switched linear control systems are stabilizable. In addition, we show that an event-driven switching stabilization strategy can be explicitly constructed.
\end{abstract}

Index Terms - Switched linear systems, reachability, stabilization, event-driven switching.

\section{INTRODUCTION}

In the last decade, there has been considerable interest in modelling, analysis and design of switched and hybrid systems (see [1, 4, 5, 9, 12] and the references therein). Switched systems deserve investigation for theoretical interest as well as for practical applications. Switching among different system structures is an essential feature of many engineering control applications such as power systems and power electronics [10, 8]. Control techniques based on switching between different controllers have also been investigated in recent years, particularly in the context of adaptive control $[6,7]$. The existence of systems that cannot be asymptotically stabilized by a single continuous feedback controller [2] also motivates us to study switched systems.

In [3], some sufficient conditions and necessary conditions were given for controllability, observability and stability for periodic switched linear control systems under the assumption that the switching sequence is fixed a priori. In this paper, we are to design not only the appropriate control inputs but also the switching strategy in order to control and/or stabilize the system. As the switching sequence is a design variable rather than fixed a priori, an

\footnotetext{
* This work was supported by National Key Project of China and National Nature Science Foundation of China.

${ }^{\dagger}$ Corresponding Author. Present address: Department of Electrical and Computer Engineering, National University of Singapore, Singapore 117576. Tel: (+65) 874 4460, Email: elesunzd@nus.edu.sg.
} 
extra degree of freedom in design is introduced.

The paper is organized as follows. Section II formulates the problem and presents preliminary analysis. A necessary condition and a sufficient condition for reachability are presented in Section III. Section IV investigates the probelm of event-driven stabilization and presents a constructive design algorithm. Finally, some concluding remarks are made in the last section.

\section{DEFINITIONS AND PRELIMINARIES}

Consider a switched linear control system given by

$$
\dot{x}(t)=A_{\alpha(x, t)} x(t)+B_{\alpha(x, t)} u_{\alpha(x, t)}(t),
$$

where $x \in \Re^{n}$ are the states, $u_{i}(t) \in \Re^{r_{i}}, i=1, \cdots, m$ are the inputs, $\alpha(x, t): \Re^{n} \times \Re^{+} \rightarrow$ $\{1,2, \cdots, m\}$ is the switching function to be designed, and matrix pair $\left(A_{k}, B_{k}\right)$ for $1 \leq k \leq m$ denotes a subsystem of (1).

For switched systems, a switching sequence is to specify when and to which subsystem one should switch at each instant of time.

Definition 1: A switching sequence is a finite or countable ordered set of pairs of time and active subsystem

$$
\left\{\left(\tau_{0}, i_{0}\right),\left(\tau_{1}, i_{1}\right), \cdots,\left(\tau_{s}, i_{s}\right)\right\}
$$

where $\tau_{0}<\tau_{1}<\cdots<\tau_{s} \leq \infty$ and $i_{j} \in\{1,2, \cdots, m\}$, for $j=0,1, \cdots, s, s \leq \infty$.

Given switched system (1) and any initial configuration $x_{0}$, the undergoing switching sequence (2) can be uniquely determined by switching function $\alpha(x, t)$, and vice-versa. The quantity relationship is

$$
\alpha(x, t)=k, \text { for } t \in \cup_{i_{j}=k}\left[\tau_{j}, \tau_{j+1}\right), k=1, \cdots, m .
$$

Definition 2: System (1) is (completely) reachable, if for any given $t_{0}, x_{0}$ and $x_{f}$, there exist a real number $t_{f}>t_{0}$, piecewise continuous control functions $u_{i}(t), t \in\left[t_{0}, t_{f}\right], i=1, \cdots, m$ and a switching function $\alpha(x, t)$, such that $x\left(t_{f}\right)=x_{f}$.

It is obvious that if one subsystem, say $\left(A_{1}, B_{1}\right)$, is controllable, then system (1) is reachable too. In this paper, we shall investigate the non-trivial situation where each subsystem 
$\left(A_{i}, B_{i}\right), i=1, \cdots, m$ is not controllable.

Denote $C_{i}=\left[B_{i}, A_{i} B_{i}, \cdots, A_{i}^{n-1} B_{i}\right]$, for $i=1, \cdots, m, \mathcal{C}_{i}=\operatorname{Im} C_{i}$, and $\operatorname{Im} B$ the subspace spanned by columns of matrix $B$. Given matrix $A$ and subspace $\mathcal{B}$, let $\Gamma_{A} \mathcal{B}$ denote the minimal invariant subspace of $\mathcal{B}$ under $A$, i.e.,

$$
\Gamma_{A} \mathcal{B}=\mathcal{B}+A \mathcal{B}+\cdots+A^{n-1} \mathcal{B} .
$$

This operation can be defined recursively as $\Gamma_{A_{1}} \Gamma_{A_{2}} \mathcal{B}=\Gamma_{A_{1}}\left(\Gamma_{A_{2}} \mathcal{B}\right)$. For clarity, define the nested subspaces as

$$
\begin{aligned}
\mathcal{V}_{1} & =\mathcal{C}_{1}+\cdots+\mathcal{C}_{m} \\
\mathcal{V}_{2} & =\Gamma_{A_{1}} \mathcal{V}_{1}+\cdots+\Gamma_{A_{m}} \mathcal{V}_{1} \\
& \vdots \\
\mathcal{V}_{n} & =\Gamma_{A_{1}} \mathcal{V}_{n-1}+\cdots+\Gamma_{A_{m}} \mathcal{V}_{n-1},
\end{aligned}
$$

Subspaces $\mathcal{V}_{1}$ and $\mathcal{V}_{n}$ are used in Theorem 1 and Theorem 2 in Section III.

Lemma 1: Given a matrix sequence $B_{1}, \cdots, B_{s}$ of the same row number $n$. If $A_{1}, \cdots, A_{s-1}$ are sufficiently close to the identity matrix $I_{n}$, then it satisfies

$$
\operatorname{rank}\left[B_{1}, \cdots, B_{s}\right] \leq \operatorname{rank}\left[B_{1}, A_{1} B_{2}, \cdots, A_{1} \cdots A_{s-1} B_{s}\right] .
$$

Proof: See Appendix A.

\section{REACHABILITY}

A simple necessary condition for reachability is summarized in Theorem 1.

Theorem 1: If switched linear system (1) is reachable, then

$$
\mathcal{V}_{n}=\Re^{n} .
$$

Proof: Suppose that system (1) is reachable and initially rest at $x\left(t_{0}\right)=0$. For arbitrarily any given $x \in \Re^{n}$, there exist $t_{f}>t_{0}$, a switching sequence $\left\{\left(\tau_{0}, i_{0}\right), \cdots,\left(\tau_{s}, i_{s}\right)\right\}$ and inputs $u_{i}(t), i=1, \cdots, m$, such that

$$
\begin{gathered}
x\left(t_{f}\right)=e^{A_{i_{s}}\left(t_{f}-\tau_{s}\right)} \cdots e^{A_{i_{1}}\left(\tau_{2}-\tau_{1}\right)} \int_{\tau_{0}}^{\tau_{1}} e^{A_{i_{0}}\left(\tau_{1}-\tau\right)} B_{i_{0}} u_{i_{0}}(\tau) d \tau \\
+\cdots+\int_{\tau_{s}}^{t_{f}} e^{A_{i_{s}}\left(t_{f}-\tau\right)} B_{i_{s}} u_{i_{s}}(\tau) d \tau=x
\end{gathered}
$$


Note that $e^{A_{i} t} \operatorname{Im} B_{i} \subset \mathcal{C}_{i}$ and $\int_{t_{1}}^{t_{2}} e^{A_{i}\left(t_{2}-\tau\right)} B_{i} u_{i}(\tau) d \tau \in \mathcal{C}_{i}$ for $i=1, \cdots, m$ and $t_{2}>t_{1}$. Accordingly,

$$
x \in \Gamma_{A_{i_{s}}} \Gamma_{A_{i_{s-1}}} \cdots \Gamma_{A_{i_{0}}} \mathcal{B}_{i_{0}}+\cdots+\Gamma_{A_{i_{s}}} \mathcal{B}_{i_{s}} \subset \mathcal{V}_{n}
$$

Since the chioce of $x$ is arbitrary, one has $\mathcal{V}_{n}=\Re^{n} . \diamond$

Remark 1: Using Theorem 1, we can easily exclude some unreachable switched linear systems. For example, system (1) with $A_{i}=0, i=1, \cdots, m$ and $\operatorname{rank}\left[B_{1}, B_{2}, \cdots, B_{m}\right]<n$ is not reachable.

Theorem 2: A sufficient condition for system (1) to be reachable is

$$
\mathcal{V}_{1}=\Re^{n}
$$

Proof: According to linear system theory, for any $i, 1 \leq i \leq m$, it is always possible to construct transformation matrices $\left(T_{i}, F_{i}, G_{i}\right)$ with $T_{i}$ and $G_{i}$ being nonsingular, such that the matrix pair

$$
\left(\hat{A}_{i}, \hat{B}_{i}\right) \stackrel{\text { def }}{=}\left(T_{i}^{-1}\left(A_{i}+B_{i} F_{i}\right) T_{i}, T_{i}^{-1} B_{i} G_{i}\right)
$$

is in the form as

$$
\hat{A}_{i}=\left[\begin{array}{cccc}
M_{1}^{i} & \cdots & 0 & M_{1, r_{i}+1}^{i} \\
& \ddots & & \vdots \\
0 & \cdots & M_{r_{i}}^{i} & M_{r_{i}, r_{i}+1}^{i} \\
0 & \cdots & 0 & M_{r_{i}+1, r_{i}+1}^{i}
\end{array}\right], \hat{B}_{i}=\left[\begin{array}{ccc}
\hat{b}_{1}^{i} & \cdots & 0 \\
& \ddots & \\
0 & \cdots & \hat{b}_{r_{i}}^{i} \\
0 & \cdots & 0
\end{array}\right],
$$

where $\left(M_{j}^{i}, \hat{b}_{j}^{i}\right), j=1, \cdots, r_{i}$ are Brunovsky normal pairs of length $k_{j}^{i}$ with $k_{1}^{i}, \cdots, k_{r_{i}}^{i}$ being the controllability indeces of subsystem $\left(A_{i}, B_{i}\right)$.

Introducing the following linear feedback control

$$
u_{i}=F_{i} x+G_{i} v_{i}, \quad i=1, \cdots, m,
$$

system (1) is transformed into

$$
\dot{x}=\left(A_{i}+B_{i} F_{i}\right) x+B_{i} G_{i} v_{i} \stackrel{\text { def }}{=} \bar{A}_{i} x+\bar{B}_{i} v_{i} .
$$


Detailed computation shows that, for any given real numbers $\tau_{0}<\tau_{1}<\cdots<\tau_{n}$, the following equations hold

$$
\operatorname{Im}\left[\int_{\tau_{0}}^{\tau_{1}} e^{\bar{A}_{i}\left(\tau_{n}-\tau\right)} d \tau \bar{B}_{i}, \cdots, \int_{\tau_{n-1}}^{\tau_{n}} e^{\bar{A}_{i}\left(\tau_{n}-\tau\right)} d \tau \bar{B}_{i}\right]=\operatorname{Im}\left[\bar{B}_{i}, \cdots, \bar{A}_{i}^{n-1} \bar{B}_{i}\right]=\mathcal{C}_{i}
$$

For clarity, let $0=t_{1}=t_{1,0}<t_{1,1}<\cdots<t_{1, n}=t_{2}=t_{2,0}<t_{2,1}<\cdots<t_{2, n}=\cdots=$ $t_{m}=t_{m, 0}<t_{m, 1}<\cdots<t_{m, n}=t_{m+1}$ be time instants for control switching to be designed. Denote

$$
\begin{aligned}
D_{i} & =\left[\int_{t_{i, 0}}^{t_{i, 1}} e^{\bar{A}_{i}\left(t_{i+1}-\tau\right)} d \tau \bar{B}_{i}, \cdots, \int_{t_{i, n-1}}^{t_{i, n}} e^{\bar{A}_{i}\left(t_{i+1}-\tau\right)} d \tau \bar{B}_{i}\right], i=1, \cdots, m, \\
E_{j} & =e^{\bar{A}_{j}\left(t_{j+1}-t_{j}\right)}, j=2, \cdots, m
\end{aligned}
$$

It follows from (10) that

$$
\operatorname{rank}\left[E_{m} \cdots E_{2} D_{1}, \cdots, E_{m} D_{m-1}, D_{m}\right]=\operatorname{rank}\left[E_{m} \cdots E_{2} C_{1}, \cdots, E_{m} C_{m-1}, C_{m}\right]
$$

Note that $\lim _{t \rightarrow 0} e^{A t} \longrightarrow I_{n}$ for any given matrix $A$. Accordingly, let $t_{m+1}$ be sufficiently small, it follows from Lemma 1 that

$$
\operatorname{rank}\left[E_{m} E_{m-1} \cdots E_{2} C_{1}, \cdots, E_{m} C_{m-1}, C_{m}\right] \geq \operatorname{rank}\left[C_{1}, \cdots, C_{m}\right]=n
$$

Equations (11) and (12) imply that

$$
\operatorname{rank}\left[E_{m} E_{m-1} \cdots E_{2} D_{1}, \cdots, E_{m} D_{m-1}, D_{m}\right]=n
$$

For system (9), consider

$$
v_{i}(t)=a_{i, j}, t_{i, j} \leq t<t_{i, j+1}, j=0, \cdots, n-1, i=1, \cdots, m
$$

where $a_{i, j}$ are variables to be determined. For any arbitrarily given $x_{0}$ and $x_{f}$, consider the equation (with unknowns $a_{i, j}, i=1, \cdots, m, j=0, \cdots, n-1$ ) given by

$$
\begin{gathered}
x_{f}-e^{\bar{A}_{m}\left(t_{m+1}-t_{m}\right)} \cdots e^{\bar{A}_{1}\left(t_{2}-t_{1}\right)} x_{0}=e^{\bar{A}_{m}\left(t_{m+1}-t_{m}\right)} \cdots e^{\bar{A}_{2}\left(t_{3}-t_{2}\right)} \int_{t_{1}}^{t_{2}} e^{\bar{A}_{1}\left(t_{2}-\tau\right)} \bar{B}_{1} v_{1}(\tau) d \tau \\
+\cdots+\int_{t_{m}}^{t_{m+1}} e^{\bar{A}_{m}\left(t_{m+1}-\tau\right)} \bar{B}_{m} v_{m}(\tau) d \tau
\end{gathered}
$$

It follows from (13) that equation (14) at least has one solution. Accordingly, system (9) is reachable, which implies reachability of $(1) \cdot \diamond$ 
In general, there is a gap between conditions (4) and (5). However, for specific classes of systems, these two conditions coincide, hence a complete characterization for reachability is available immediately. The simplest example is the non-switched systems $(m=1)$, for which Theorem 1 and Theorem 2 together leads to the well known solution for controllability [11]:

$$
\mathcal{B}_{1}+A_{1} \mathcal{B}_{1}+\cdots+A_{1}^{n-1} \mathcal{B}_{1}=\Re^{n} .
$$

Another example is the system with $A_{1}=A_{2}=\cdots=A_{m}$, i.e., a nominal (non-switching) system with multiple controllers. Consequently, condition (5) is a necessary and sufficient condition for this class of systems.

For low-dimensional switched linear systems, the gap between conditions (4) and (5) can be filled up through analyzing all possible switching sequences.

Corollary 1: For system (1) with $n=3, m=2$, a necessary and sufficient condition for reachability is

$$
\mathcal{V}_{3}=\Re^{3}
$$

Proof: See Appendix B.

\section{EVENT-DRIVEN FEEDBACK STABILIZATION}

In this section, let us investigate the problem of stabilization for switched linear control systems. In the sequel, we make two assumptions: (i) each subsystem $\left(A_{i}, B_{i}\right)$ is not stabilizable for $i=1, \cdots, m$, and (ii) system (1) satisfies relationship (5).

Define a sequence of subspaces of $\Re^{n}$ described by:

$$
\mathcal{W}_{k}=\sum_{i_{1}, \cdots, i_{k-1}=1}^{m}\left(\cap_{j \notin\left\{i_{1}, \cdots, i_{k-1}\right\}} \mathcal{C}_{j}\right), \quad k=1, \cdots, m,
$$

It is obvious that $\mathcal{W}_{1} \subset \mathcal{W}_{2} \subset \cdots \subset \mathcal{W}_{m}=\sum_{i=1}^{m} \mathcal{C}_{i}=\Re^{n}$.

A basis of $\Re^{n}$ can be constructed according to the following procedure. First, choose a group of base vectors $\gamma_{1}, \cdots, \gamma_{s_{1}}$ in $\mathcal{W}_{1}$. Then, expand them to $\gamma_{1}, \cdots, \gamma_{s_{1}}, \gamma_{s_{1}+1}, \cdots, \gamma_{s_{2}}$ which form a basis of $\mathcal{W}_{2}$. Continuing this process, we finally find a basis $\gamma_{1}, \cdots, \gamma_{n}$ of $\mathcal{W}_{m}=\Re^{n}$. Define $T=\left[\gamma_{1}, \cdots, \gamma_{n}\right]$.

Let $z(t)=T^{-1} x(t)$. System (1) can be re-written as

$$
\dot{z}=T^{-1} A_{\alpha}(x, t) T z+T^{-1} B_{\alpha}(x, t) u_{\alpha}(x, t) .
$$


Suppose $\operatorname{dim} \mathcal{C}_{i}=k_{i}$ and $\left\{z_{i_{1}}, \cdots, z_{i_{k_{i}}}\right\} \in \mathcal{C}_{i}$ with $i_{1}<i_{2}<\cdots<i_{k_{i}}$. Let

$$
\begin{aligned}
y_{i} & =\left[z_{i_{1}}, \cdots, z_{i_{k_{i}}}\right]^{T}, \quad i=1, \cdots, m, \\
y^{i} & =\left[z_{1}, \cdots, z_{i_{1}-1}, z_{i_{1}+1}, \cdots, z_{i_{2}-1}, \cdots, z_{i_{k_{i}}+1}, \cdots, z_{n}\right]^{T}, \quad i=1, \cdots, m,
\end{aligned}
$$

Let $\sigma_{i, 1}, \cdots, \sigma_{i, \Gamma_{i}}$ denote the real parts of eigenvalues of $A_{i}$ corresponding to the uncontrollable mode of system $\left(A_{i}, B_{i}\right)$. From assumption (i) made above, it follows that $\sigma_{i}=\max \left\{\sigma_{i, 1}, \cdots, \sigma_{i, \Gamma_{i}}\right\} \geq 0$, for $i=1, \cdots, m$. Define $\sigma_{\max }=\max \left\{\sigma_{i}: i=1, \cdots, m\right\}$.

Fix a $r>m \sigma_{\max }+1$. It is routine to construct feedback gain matrices $F_{i}, i=1, \cdots, m$, such that each of the real parts of eigenvalues of $A_{i}+B_{i} F_{i}$ corresponding to the controllable mode of $\left(A_{i}, B_{i}\right)$ is less than $-r$. Applying feedback transformations

$$
u_{i}(t)=F_{i} x(t), \quad i=1, \cdots, m,
$$

equation (16) becomes

$$
\dot{z}=T^{-1}\left(A_{\alpha}+B_{\alpha} F_{\alpha}\right) T z .
$$

Define the norm of a vector $\zeta=\left[\zeta_{1}, \cdots, \zeta_{l}\right]^{T} \in \Re^{l}$ as

$$
\|\zeta\|=\|\zeta\|_{\infty}=\max \left\{\left|\zeta_{i}\right|: i=1, \cdots, l\right\} .
$$

Note that $y_{i}$ is in the controllability subspace of subsystem $\left(A_{i}, B_{i}\right)$, accordingly, the real parts of the corresponding poles are less than $-r$. Therefore, if $\alpha(x, t)=i$ for $t \in\left[t_{1}, t_{2}\right]$, then

$$
\left\|y_{i}\left(t_{2}\right)\right\| \leq c_{i} e^{-r\left(t_{2}-t_{1}\right)}\left\|y_{i}\left(t_{1}\right)\right\|
$$

for some positive constant $c_{i}$. Similarly, for any $\sigma$ with $\sigma>\sigma_{\max }$, we have

$$
\begin{aligned}
& \left\|y_{i}\left(t_{2}\right)\right\| \leq d_{i} e^{\sigma\left(t_{2}-t_{1}\right)}\left\|y_{i}\left(t_{1}\right)\right\|, \quad i=1, \cdots, m \quad \forall t_{2}>t_{1} \\
& \left\|z\left(t_{2}\right)\right\| \leq d_{i} e^{\sigma\left(t_{2}-t_{1}\right)}\left\|z\left(t_{1}\right)\right\|
\end{aligned}
$$

for some positive constant $d_{i}$.

Fix a $\sigma$ with $\sigma_{\max }<\sigma<\sigma_{\max }+\frac{1}{m}$ and correspondingly $d_{i}$ for each $i=1, \cdots, m$. Let $c=\max \left\{c_{1}, \cdots, c_{m}, d_{1}, \cdots, d_{m}, 1\right\}$. Fix a positive real number $\tau>\frac{(m+1) \ln c}{r-m \sigma}$. We are now ready to formulate an event-driven switching strategy for system (20). 
For any given initial state $z_{0}=z\left(t_{0}\right)$, there must exist an integer $k_{0}, 1 \leq k_{0} \leq n$ such that $\left\|y_{k_{0}}\left(t_{0}\right)\right\|=\left\|z_{0}\right\|$. Define recursively the switching pairs $\left(t_{i}, k_{i}\right), i=1,2, \cdots$ to satisfy the equations given by

$$
\begin{aligned}
& t_{i}=\inf \left\{t: t \geq t_{i-1}+\tau \text { and }\left\|y_{k_{i-1}}(t)\right\| \leq\left\|y^{k_{i-1}}(t)\right\|\right\} \\
& \left\|y_{k_{i}}\left(t_{i}\right)\right\|=\left\|z\left(t_{i}\right)\right\|
\end{aligned}
$$

where $\inf \emptyset=+\infty$ with $\emptyset$ standing for the empty set. If more than one $k_{i}$ 's satisfy equation (24), then just pick anyone among them. Let the switching sequence be

$$
\left\{\left(t_{0}, k_{0}\right),\left(t_{1}, k_{1}\right), \cdots,\left(t_{j}, k_{j}\right), \cdots\right\}
$$

Accordingly, the switching function $\alpha(x, t)$ is

$$
\alpha(x, t)=k_{i}, \quad \text { if } t_{i} \leq t<t_{i+1}
$$

Theorem 3: For switched system (1) satisfying (5), consider state feedback (19). If the event-driven switching function is given by (26), then the closed-loop system is asymptotically stable.

Proof: For any fixed $j \geq 0$, consider the interval $\left[t_{j}, t_{j+m+1}\right]$. We are to prove that the rate of convergence of $\|z(t)\|$ in this interval is higher than $\delta \stackrel{\text { def }}{=} \frac{(r-m \sigma) \tau-(m+1) \ln c}{(m+1) \tau}$. As a consequence, the closed-loop system is asymptotically stable.

Denote $\Lambda=\left\{l: j \leq l \leq j+m, t_{l+1}-t_{l}>\tau\right\}$. From (21) and (22), it follows that

$$
\left\|z\left(t_{l+1}\right)\right\|\left\{\begin{array}{l}
=\left\|y_{k_{l}}\left(t_{l+1}\right)\right\| \leq c e^{-r h_{l}}\left\|z\left(t_{l}\right)\right\| \quad \text { if } l \in \Lambda \\
\leq c e^{\sigma h_{l}}\left\|z\left(t_{l}\right)\right\|=c e^{\sigma \tau}\left\|z\left(t_{l}\right)\right\| \quad \text { else }
\end{array} \quad j \leq l \leq j+m\right.
$$

where $h_{l}=t_{l+1}-t_{l}, l=j, \cdots, j+m$.

Suppose $\Lambda \neq \emptyset$, then from (27), we have

$$
\left\|z\left(t_{j+m+1}\right)\right\| \leq c^{m+1} e^{\left(\rho \sigma \tau-r \sum_{j \in \Lambda} h_{j}\right)}\left\|z\left(t_{j}\right)\right\| \leq e^{-\delta\left(t_{j+m+1}-t_{j}\right)}\left\|z\left(t_{j}\right)\right\|
$$

where $\rho$ is the number of elements of set $\left\{l: j \leq l \leq j+m, h_{l}=\tau\right\}$. The latter inequality of (28) holds due to $\delta<\frac{r \sum_{j \in \Lambda} h_{j}-(m+1) \ln c-\rho \sigma \tau}{t_{j+m+1}-t_{j}}$. 
On the other hand, if $\Lambda=\emptyset$, then $h_{l}=\tau, l=j, \cdots, j+m$. Because $i_{l} \in\{1, \cdots, m\}$ for $l=j, \cdots, j+m$, there must exist integers $l_{1}, l_{2}, j \leq l_{1}<l_{2} \leq j+m$, such that $k_{l_{1}}=k_{l_{2}}$. From (27) and (22), it follows that

$$
\begin{aligned}
\left\|z\left(t_{j+m+1}\right)\right\| & \leq c^{j+m+1-l_{2}} e^{\left(j+m+1-l_{2}\right) \sigma \tau}\left\|z\left(t_{l_{2}}\right)\right\|=c^{j+m+1-l_{2}} e^{\left(j+m+1-l_{2}\right) \sigma \tau}\left\|y_{k_{l_{2}}}\left(t_{l_{2}}\right)\right\| \\
& \leq c^{j+m-l_{1}} e^{\left(j+m-l_{1}\right) \sigma \tau}|| y_{k_{l_{2}}}\left(t_{l_{1}+1}\right)\left\|\leq c^{j+m+1-l_{1}} e^{\left(j+m-l_{1}\right) \sigma \tau-r \tau}\right\| y_{k_{l_{1}}}\left(t_{l_{1}}\right) \| \\
& =c^{j+m+1-l_{1}} e^{\left(j+m-l_{1}\right) \sigma \tau-r \tau}\left\|z\left(t_{l_{1}}\right)\right\| \leq c^{m+1} e^{m \sigma \tau-r \tau}\left\|z\left(t_{j}\right)\right\| \\
& =e^{-\delta(m+1) \tau}\left\|z\left(t_{j}\right)\right\|=e^{-\delta\left(t_{j+m+1}-t_{j}\right)}\left\|z\left(t_{j}\right)\right\| . \quad \diamond
\end{aligned}
$$

Remark 2: Note that if $A_{1}=A_{2}=\cdots=A_{m}$ in (1), then stabilization of a switched system (1) can be seen as stabilization of a linear system via multi-controller switching. In this scheme, two problems at different levels involve interactively: at the low-level, we need to choose a number of candidate controllers, and at the high-level, we are to determine a suitable event-driven switching strategy (the supervisor) to ensure stability of the overall system. In the context of adaptive control of linearly parameterized systems, this eventdriven switching scheme is termed as 'logic-based switching and control' [6, 7].

Remark 3: Note that for each subsystem, the convergent rate of the controllable subdynamics dominates divergent rate of uncontrollable sub-dynamics. As a consequence, periodic switching with large dwell time could also lead to asymptotic stability. That is, for sufficiently large dwell time $\tau$, define periodic switching sequence as

$$
\{(0,1),(\tau, 2), \cdots,((m-1) \tau, m),(m \tau, 1),((m+1) \tau, 2), \cdots,((2 m-1) \tau, m), \cdots\}
$$

then the closed-loop system of (1), (19) and (29) is asymptotically stable. 
Example 1: Let $n=5, m=3$, and

$$
\begin{gathered}
A_{1}=\left[\begin{array}{lllll}
0 & 0 & 0 & 1 & 0 \\
0 & 1 & 0 & 0 & 0 \\
0 & 0 & 0 & 0 & 0 \\
0 & 0 & 0 & 0 & 0 \\
0 & 0 & 0 & 0 & 1
\end{array}\right], B_{1}=\left[\begin{array}{l}
0 \\
0 \\
0 \\
1 \\
0
\end{array}\right] ; A_{2}=\left[\begin{array}{lllll}
0 & 1 & 0 & 0 & 0 \\
0 & 0 & 0 & 0 & 0 \\
0 & 0 & 0 & 0 & 0 \\
0 & 0 & 0 & 1 & 0 \\
0 & 0 & 0 & 0 & 2
\end{array}\right], B_{2}=\left[\begin{array}{lll}
0 & 0 \\
1 & 0 \\
0 & 1 \\
0 & 0 \\
0 & 0
\end{array}\right] ; \\
A_{3}=\left[\begin{array}{ccccc}
1 & 0 & 0 & 0 & 0 \\
0 & 0 & -1 & 0 & 0 \\
0 & 0 & 0 & 1 & 0 \\
0 & 0 & 0 & 0 & 0
\end{array}\right], B_{3}=\left[\begin{array}{l}
0 \\
0 \\
0 \\
0 \\
1
\end{array}\right] .
\end{gathered}
$$

One may verify that $\mathcal{V}_{1}=\Re^{5}$. According to Theorem 3 , this system is stabilizable.

By fixing $r=10$ and $\tau=0.2$, a stabilizing state feedback and switching strategy can be obtained accordingly. Let us consider the initial state given by

$$
x(0)=[-2.0452,2.0757,-0.7796,-2.7625,0.6311] .
$$

Figure 1 shows the convergence of the states, while Figure 2 gives the corresponding switching sequence. As shown in Figure 2, neither the sequence of active subsystem nor the duration on each subsystem is periodic.

As pointed in Remark 3, for sufficiently large dwell time, a periodicly switching strategy also result in a stable closed-loop system. Figure 3 shows the convergence of the states with dwell time $\tau=0.3$. An intensive simulation study exhibits that, for any dwell time smaller than 0.25 , the state trajectories of the closed-loop system diverge to infinity at an exponential rate.

\section{CONCLUSION}

Some reachability and stabilization results have been presented for switched linear control systems. For reachability, a necessary condition and a sufficient condition have been presented. In particular, for third-order systems, a necessary and sufficient condition has been 
obtained. Under mild assumptions, an event-driven switching strategy has been proposed to ensure asymptotic convergence of the system.

\section{ACKNOWLEDGEMENT}

The authors would like to thank S. S. Ge and the reviewers for their help and insightful comments for further improving the quality of this work.

\section{References}

[1] M. S. Branicky, "Multiple Lyapunov functions and other analysis tools for switched and hybrid systems," IEEE Trans. Automat. Contr., vol.43, pp.475-482, 1998.

[2] R. W. Brockett, "Asymptotic stability and feedback stabilization," in Differential Geometric Control Theory, R. W. Brockett et al, eds., Boston: Birkhauser, 1983, pp. $181-191$.

[3] J. Ezzine and A. H. Haddad, "Controllability and observability of hybrid systems," Int. J. Contr., vol.49, pp.2045-2055, 1989.

[4] W. P. Dayawansa and C. F. Martin, "A converse Lyapunov theorem for a class of dynamical systems undergo switching," IEEE Trans. Automat. Contr., vol.44, pp.751760, 1999.

[5] D. Liberzon and A. S. Morse, "Basic problems in stability and design of switched systems," IEEE Contr. Syst. Mag., vol. 19, no. 5, pp. 59-70, 1999.

[6] A. S. Morse, "Supervisory control of families of linear set-point controllers- Part 1: exact matching," IEEE Trans. Automat. Contr., vol.41, pp. 1413-1431, 1996.

[7] K. S. Narendra and J. Balakrishnan, "Adaptive control using multiple models," IEEE Trans. Automat. Contr., vol.42, pp. 171-187, 1997.

[8] H. Sira-Ramirez, "Nonlinear P-I controller design for switch mode dc-to-dc power converters," IEEE Trans. Circuits Syst., vol. 38, pp. 410-417, 1991. 
[9] M. A. Wicks, P. Peleties and R. A. DeCarlo, "Switched controller synthesis for the quadratic stabilization of a pair of unstable linear systems," European J. Contr., vol. 4, pp. 140-147, 1998.

[10] S. M. Williams and R. G. Hoft, "Adaptive frequency domain control of PPM switched power line conditioner," IEEE Trans. Power Electron, vol. 6, pp. 665-670, 1991.

[11] W. M. Wonham, Linear Multivariable Control - A Geometric Approach. Berlin: Spinger-Verlag, 1974.

[12] H. Ye, A. N. Michel and L. Hou, "Stability theory for hybrid dynamical systems," IEEE Trans. Automat. Contr., vol. 43, pp.461-474, 1998.

\section{APPENDIX A}

Proof of Lemma 1: First, let us consider the case where $s=2$.

Denote $A_{1}=I_{n}+E$. Then $\left[B_{1}, A_{1} B_{2}\right]=\left[B_{1}, B_{2}+E B_{2}\right]$. Choosing a nonsingular submatrix $G$ with maximal rank in $\left[B_{1}, B_{2}\right]$, we denote the corresponding submatrix of $\left[B_{1}, A_{1} B_{2}\right]$ as $G^{\prime}=G+\Delta$. Because each element of $\Delta$ is sufficiently small, $G^{-1} G^{\prime}=$ $I_{n}+G^{-1} \Delta$ is strictly diagonal dominant and, subsequently, $G^{\prime}$ is nonsingular. This implies that $\operatorname{rank}\left[B_{1}, A_{1} B_{2}\right] \geq \operatorname{rank}\left[B_{1}, B_{2}\right]$.

For the case when $s>2$, one may obtain recursively that

$$
\begin{gathered}
\operatorname{rank}\left[B_{1}, A_{1} B_{2}, \cdots, A_{1} \cdots A_{s-1} B_{s}\right]=\operatorname{rank}\left[B_{1}, A_{1}\left[B_{2}, A_{2} B_{3}, \cdots, A_{2} \cdots A_{s-1} B_{s}\right]\right] \\
\geq \operatorname{rank}\left[B_{1}, B_{2}, A_{2} B_{3}, \cdots, A_{2} \cdots A_{s-1} B_{s}\right] \geq \cdots \geq \operatorname{rank}\left[B_{1}, \cdots, B_{s}\right] .
\end{gathered}
$$

\section{APPENDIX B}

Proof of Corollary 1: The necessity follows directly from Theorem 1. We only need to prove the sufficiency.

By Theorem 2, we may assume, without loss of generality, that rank $\mathcal{V}_{1}<3$. It can be verified that the case of $\operatorname{rank} B_{1}=\operatorname{rank} B_{2}=2$ contradicts (15). This means that one of the following cases must hold. 
(i) $\operatorname{rank} B_{1}=\operatorname{rank} B_{2}=1, \operatorname{rank}\left[B_{1}, B_{2}\right]=2$;

(ii) $\operatorname{rank} B_{1}=1, \operatorname{rank} B_{2}=2, \operatorname{Im} B_{1} \subset \operatorname{Im} B_{2}$;

(iii) $\operatorname{rank} B_{1}=2, \operatorname{rank} B_{2}=1, \operatorname{Im} B_{2} \subset \operatorname{Im} B_{1}$; and

(iv) $\operatorname{rank}\left[B_{1}, B_{2}\right]=1$.

For Case (i), it must true that $\operatorname{rank}\left[B_{1}, B_{2}, A_{2} B_{1}, A_{1} B_{2}\right]=3$. Without loss of generality, we assume that $\operatorname{rank}\left[B_{1}, B_{2}, A_{2} B_{1}\right]=3$. It can be verified that there exist $0=t_{0}<t_{1}<t_{2}$ such that

$$
\operatorname{rank}\left[e^{A_{2}\left(t_{2}-t_{1}\right)} \int_{t_{0}}^{t_{1}} e^{A_{1}\left(t_{1}-\tau\right)} d \tau B_{1}, \int_{t_{1}}^{t_{2}} e^{A_{2}\left(t_{2}-\tau\right)} d \tau B_{2}, B_{1}\right]=3
$$

Let $t_{3}>t_{2}$ with $t_{3}-t_{2}$ sufficiently small. Let the switching sequence and inputs be $\left\{\left(t_{0}, 1\right),\left(t_{1}, 2\right),\left(t_{2}, 1\right)\right\}$ and

$$
u_{1}(t)=a_{1}, t_{0} \leq t<t_{1}, u_{2}(t)=a_{2}, t_{1} \leq t<t_{2}, u_{1}(t)=a_{3}, t_{2} \leq t<t_{3}
$$

respectively. This control strategy steers the system from an arbitrary given initial configuration $x\left(t_{0}\right)=x_{0}$ to any given state $x_{f}$ at $t_{3}$ by appropriately choosing $a_{1}, a_{2}$ and $a_{3}$. (Cf. Proof of Theorem 2).

Noting that Cases (ii) and (iii) are symmetric, we only need to consider Case (ii). It follows from (15) that $\operatorname{rank}\left[B_{2}, A_{1} B_{2}\right]=3$. Thus, $B_{2}$ can be denoted as (up to some state feedback) $\left[B_{1}, b_{2}\right]$. Let $u_{2}(t)=\left[\bar{u}_{2}(t), 0\right]^{T}$, then it turns into Case (i) for conclusion.

Case (iv) can be proved similarly as for Case (i) and the details are omitted. 


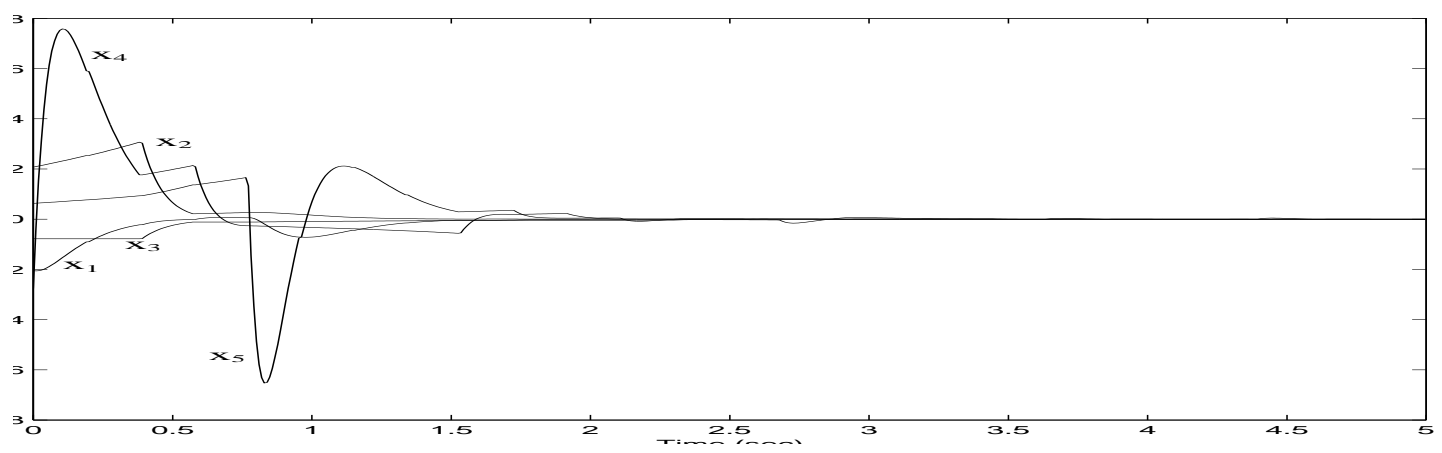

Figure 1: State trajectories under event-driven switching strategy

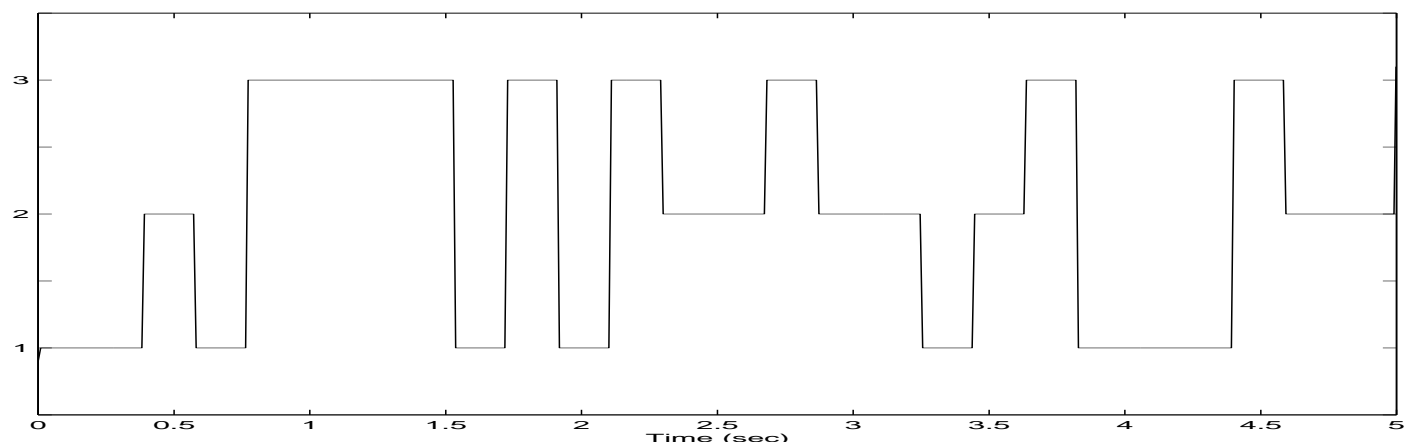

Figure 2: Switching path under event-driven switching strategy

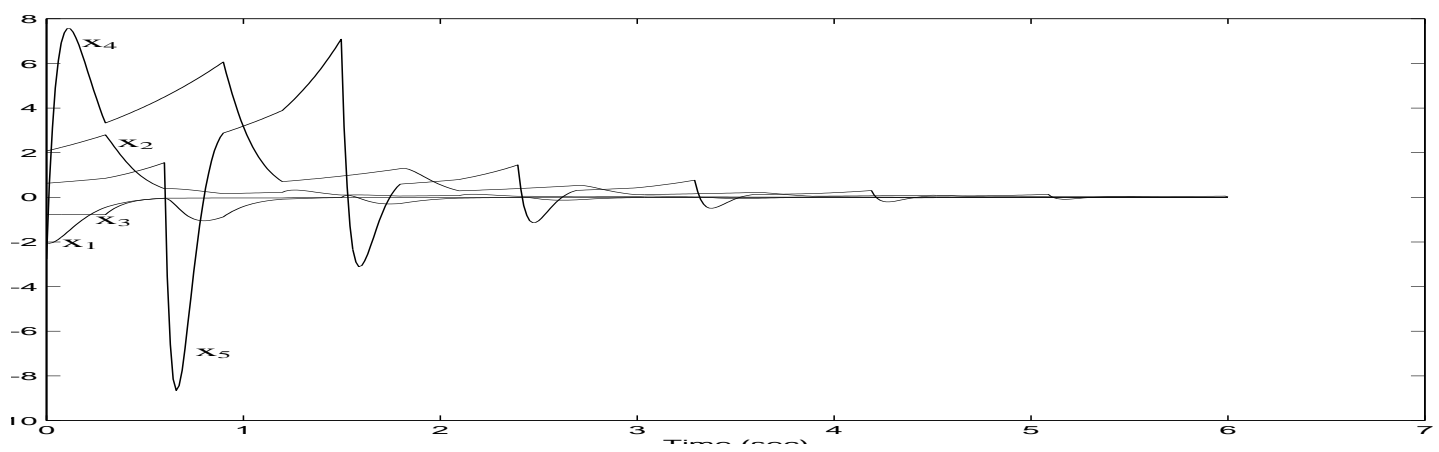

Figure 3: State trajectories under periodic switching strategy 